KONRAD RUDNICKI

\title{
NEUROBIOLOGICAL BASIS FOR EMERGENCE OF NOTIONS
}

\section{Introduction}

Even though psychology and logic share several topics of common interest, they operate within different paradigms and therefore encounter difficulties in mutual communication of their advances. One of the most dire problems shared by both is the question of acquisition and structure of notions. Both of these issues have been minutely addressed from perspective of logic in the previous article entitled "Two procedures expanding a linguistic competence" (Łukowski, 2015). The theory proposed there for development of linguistic competence and ontogenesis of notions, has significant impact on foundations of semantics. Its claims could not be made if not for several neuroscientific insights into functioning of the central nervous system that resulted in refining the models of cognitive processes. It is apparent that emergence of notions in an individual is a direct product of brain activity. Even though philosophers still contest the character of relationship between neural function and psychological phenomena, it is no longer controversial in science that the structure of the first determines the structure of the latter. Following that, any theory expressed in language of psychology or philosophy should comply with inferences concerning the architecture of thinking that is derived from neurophysiological data. Although there is a vast amount of information concerning neurobiological correlates of psychological phenomena some of them are of special interest in the discourse on notions and the meaning of words. This chapter will focus especially on biological substructure underlying vagueness, generality, dynamicity and temporality of notions. 


\section{Basic concepts}

Every neuronal network, be it artificial or natural, requires constant flow of new inputs (information) to properly function and produce useful output. In case of the human brain inputs are provided by receptors of sensory systems. Contrary to the popular belief, humans have more than five senses. Their actual number is estimated to be around twenty, depending on adopted definition of sense. When matter of learning is raised, it is customary in philosophy to concentrate the argument around visual perception. That practice is not groundless as majority of sensory information received by human brain is visual. $10^{10} \mathrm{bits}$ of information is coded by retina each second. Considering that human cortical, sensory network is approximately convergent (Foxe, Schroeder, 2005), only a fraction of the mentioned amount of information reaches cerebral cortex. Around $10^{4} \mathrm{bits} / \mathrm{sec}$ ultimately reaches the fourth layer of primary visual cortex (V1) (Raichle, 2010). $\mathrm{V} 1$ processes visual stimuli at the most rudimentary level. Its cells are sensitive to the most basic kinds of stimuli, for instance straight lines or dots, with respect to their spatial orientation. After preprocessing, signal is propagated to cortical areas where neurons respond to more complex sets of properties. In terms of notion creation it is crucial to note that information, coded by higher-level cortical networks, is derived from activity associated with the simplest stimuli. Pivotal role in the transition between initial processing and emergence of meaning is played by the association areas that integrate information from different modalities. It is still a matter of debate whether the most important role in the generation of conscious thought and concepts is played by some specialized areas or interconnectivity along with the interplay of a whole network. However, the activity of these association areas is definitely essential to this problem (Freeman, 1998). Apart from cortices on the borders between lobes these areas include thalamus and cerebellum, while a central position in that network is occupied by prefrontal cortex. At all stages of the information processing, back-propagation of activity from higher to lower areas is observed. This reafference process is partially responsible for a currently established paradigm of constructivism in regard to perception. In simple words: people do not perceive things purely by means of an incoming stimuli, but as they expect/understand/reckon them to be. The most famous process associated with this phenomenon is called priming. It would appear that when primarily prompted with stimulus from a given category, people are able to recognize further stimuli from that category faster than from other categories. This process

\section{O) COGNITIVE SCIENCE}


have been demonstrated in many different kinds of material, ranging from verbal (McNamara, 2005) to emotional (Hart et al., 2010). This concept leads to the second important principle of brain functioning.

While activation triggered by stimuli is the first component to be considered in generation of a single state of the network, there are others to be accounted for. Equal importance is held by pre-existing architecture of the neural network, determined by biological development and all information processed in the past (Freeman, 2004). All neural networks are shaped through learning processes governed by synaptic plasticity. Exemplary mechanisms of synaptic plasticity are long term potentiation and long term depression. The first constitutes strengthening of the synaptic connection between two neurons in response to repeated occurrence of action potentials, while outcome of the latter is opposite. In conjunction, these two are among the most prominent processes shaping architecture of the neuronal network (Tsumoto, 1992). There are multiple more mechanisms that co-determine neuronal web; all of them utilize exogenous or endogenous stimuli as triggers, which prepares the system to recept future ones. Following from mentioned principles, a meaningful state is understood as a dynamical activity pattern evoked by stimulus, constructed with respect to pre-existing state of the network, partially incorporating stimulus properties (Freeman, 2004). Activity that constitutes these states can be further convergently processed to produce their incomplete representation in the form of an expression (verbal if necessary) that can be communicated in order to elicit similar state in another human being. Incompleteness of linguistic representation stems from convergent architecture of processing which determines that verbalization codes less information than neural state. Even though these states are highly dynamical and difficult to grasp, it turns out that they occur in discrete stages, where each stage begins with a transition of the whole network activity to a new spatiotemporal pattern (Freeman, 1998). What is also worth mentioning is the fact that each past state co-determines future states through management of new stimuli influx by means of behavior, further expanding the importance of constructivism in perception. It might appear that some special properties are required from each meaningful state to discern them from non-meaningful states. It would however be controversial on the grounds of biology to contest the presence of meaning even in states unrelated to language (Atlan, Cohen, 1998), since what constitutes a meaning is a response of an organism to any representation with potential informational load. Essentially, for the immune system an antigen is information-bearing 
representation, which through interaction with immune cells conveys that information and elicits immune response specifically dependent on the type of antigen in question. Be that as it may, when the problem of notions is concerned, language is necessary and crucial.

Neurolinguistic studies already partially described networks engaged in language processing. Most commonly localized in the left hemisphere, collectively called perisylvian cortex, these areas include inferior frontal cortex, superior temporal cortex, inferior parietal cortex, operculum and insula. Any damage to these areas produces deficits in language production or understanding (aphasias). These areas are densely connected to each other, receive information and partially overlap with higher-order multimodal areas (Catani, Jones, 2005), forming strong links between language and action (Bedny, Caramazza, 2011) what is vital in regard to generation of meaningful states. Embodied cognition view even suggested that experience of meaning was embedded in the activation of sensory-motor control systems (Hauk et al., 2004). However, it was experimentally proven that the activation of the modal-specific circuits is not absolutely necessary for comprehension, but is instead involved in learning and intentional action, along with networks responding preferentially to the semantic aspect of the language (Bedny, Caramazza, 2011; Chatterjee, 2010). Nonetheless, it is established that repeated perception of objects from the same category creates a typical network activity pattern which emerges from the generalization of properties of multiple exemplars, and as such can be approximately extracted through statistical analysis. That pattern should be understood in terms of attractor basin for dynamical neural system (Duch, Dobosz, 2011), not as set of features of the prototypical object around which a category is layered (Foo, Low, 2008). Note that different networks present in the brain code different levels of abstraction and not in all cases the activation of higher-level areas is necessary (Binder, Desai, 2011). Aforementioned perisylvian areas seem to categorize information in an abstract form, partially corresponding to linguistic categories such as: nouns, verbs, events, closed-class words, etc. (Martin, 2007; Price, 1998). Figure 1 schematically depicts axonal connections between all three mentioned networks. It is yet important to note that the process of sentence comprehension is by all means not a simple addition of single word meanings corrected for the context. It involves numerous streams that process information in regard to: abstract semantic representations, syntactic structures and extra-linguistic sources of information. These

\section{2) COGNITIVE SCIENCE}


streams are specifically sensitive to hundreds of different sentence properties. Their functioning is studied with evoked potentials, functional magnetic resonance and magnetoencephalography (Panizza, 2012).

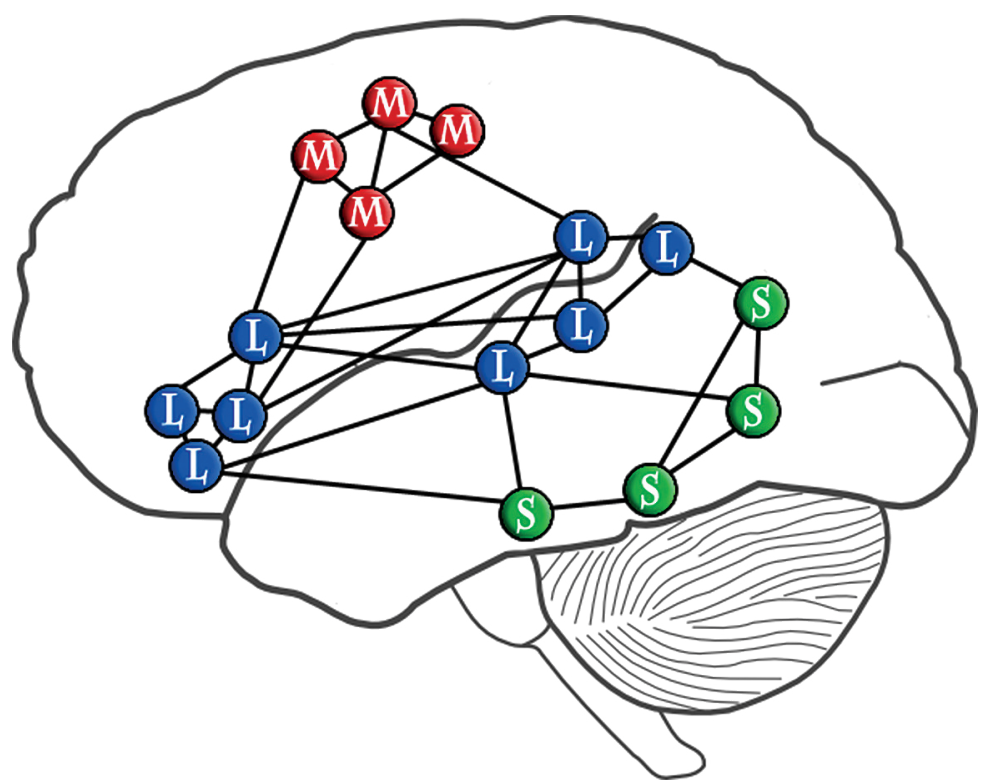

Fig. 1. Schematical visualization of connections between: $L$ - perisylvian cell assemblies, $\mathrm{R}$ - semantic circuits in the inferior-temporal object perception stream and $\mathrm{M}$ - fronto-central motor systems (Pulvermüller, 2012)

Even though spatial and temporal resolution of methods currently used for the visualization of brain activity is still lacking, neuroscientists resiliently push forward our understanding of cognitive networks by experimentally extracting crucial components of thought. Only recently, a way to communicate with people in a vegetative state, by means of functional magnetic resonance, was found. Experimenters instructed patients to imagine playing tennis if they wanted to answer yes, or imagine their old apartment if they wanted to answer no, even though there was no guarantee that patients even heard the question. It turned out that approximately $20 \%$ of patients in vegetative state were conscious and able to answer questions by imagining concepts while experimenters were able to decode their answers from patterns of brain activity (Owen et al., 2006). Even extremely complex notions are currently described with their substantial neural 
correlates, as it would appear that the same parts of cortical network are active in experience of beauty when people view classical art and when mathematicians see allegedly beautiful equations (Zeki et al., 2014). This exemplifies contemporary, spectacular advancements in fields investigating neural activation underlying the emergence of complex notions.

\section{Dynamicity and zone of proximal development}

The acquisition of linguistic competence, a process responsible for emergence of notions was said to be "controlled from outside and depend on the regular provision of new information about the correct use of words" (Eukowski, 2015). Neuronal networks require frequent stimulation of all their compartments, otherwise connections between cells will deteriorate and stored information will vanish. It is apparent though, that networks filter all incoming information, based on its relevance. Otherwise chaotic influx of stimuli would be comparable to a neuronal noise (resting-state) and network would take random shape. Aside from the attentional processes responsible for constant governance of explorative behavior, social factors influence the magnitude of each new information acquired by the brain. In dialogues presented in the previous chapter, role of the father was to ascribe the weights to information in the process of ostensive defining, in which neuronal network was engaged. Through reafference, new information, about "buildings" provided by visual system, with addition of verbal information provided by auditory system, was graded as crucial due to the presence of the father - who in this case represented the zone of proximal development. As a result, cells and connections that might have been associated with inessential properties of the notion will deteriorate, while those essential will be provided with means of strengthening (for instance, neurotrophins). Moreover, management of the attributed importance of information includes control of the function of similarity. Through feedback in social situations people learn what volume of change in perception calls for change in linguistic description.

Zone of proximal development illustrates the environment and its conditions in which a child feels safe and has the ability to explore. In the course of development, shape of the zone changes and impact size of different elements varies (for example in adolescence emphasis shifts from parents to peers). For that reason even though meaning of a word is an incidental neural phenomenon it is 
co-determined by the community we live in. Closely related to that concept is the dynamicity of notions. Following from the fact that each state of the network changes the network itself, it is evident that there can never be two situations in which a notion is understood in exactly the same way. Only after reduction to its linguistic representation which is an incomplete derivative of the incidental mental state, it might seem that notion has its complete and definite description.

\section{Model examples and generality}

In analysis of neural dynamics, the model example for a given word would be detectable if no further stimulus is provided besides the word itself. It would then be possible to describe it as "a prototype vector or a specific distribution of semantic layer activations” (Duch, Dobosz, 2011). Linguistic similarity between words can be assessed in many different ways, and only some of them bear relationship to architecture of the brain's semantic system (Carlson et al., 2014). Nevertheless, it would appear that linguistically similar words have similar attractor basins, which are a way to describe neural activity dynamics. For instance, concrete words require a lot more activity from the network than abstract words, since more properties are required to be represented. Consequently, concrete words reach their attractor basins faster (Duch, Dobosz, 2011). "Decision" of the network (the outcome of processing expressed i.e. as a verbalization) on the membership of the stimuli to one of the categories stems from the degree of similarity of the activation elicited by that stimuli to a prototype vector. The similarity is however not judged, but rather is the cause for a certain outcome (further neural activity). Even though these processes are highly dynamic and temporal, it would seem that access to their shape is possible, since it is possible to distinguish instantaneous, recurring, stable states of synchronization (Fingelkurts, Fingelkurts, 2001). Given repeated exposition to various uses of the word "temple", it comes to no surprise that information coded by a model example in a form of neural activation yields no resemblance to a description of any particular type of temple. Similarly, in the research by Zeki et al. (2014) there is no attempt to define "beauty", since what is characteristic for all beautiful things might be impossible to articulate, even though we know that it is processed specifically by medial orbitofrontal cortex. That is why the generality of notions arises from inherent properties of information processing and language acquisition (fig. 2). 
It follows that the sufficient similarity is determined through production of the same communicational outcome (i.e. a word) in every particular instance separately. After being perceived by another person (with his/her own architecture of the neural network), it gives raise to the partiality of a given content of the expression. That phenomenon falls in line with several other manifestations of the economics in cognitive processes. It would require vast amounts of time and resources from the network or might even be impossible, to produce a language without convergent processing and partial sacrifice of information.

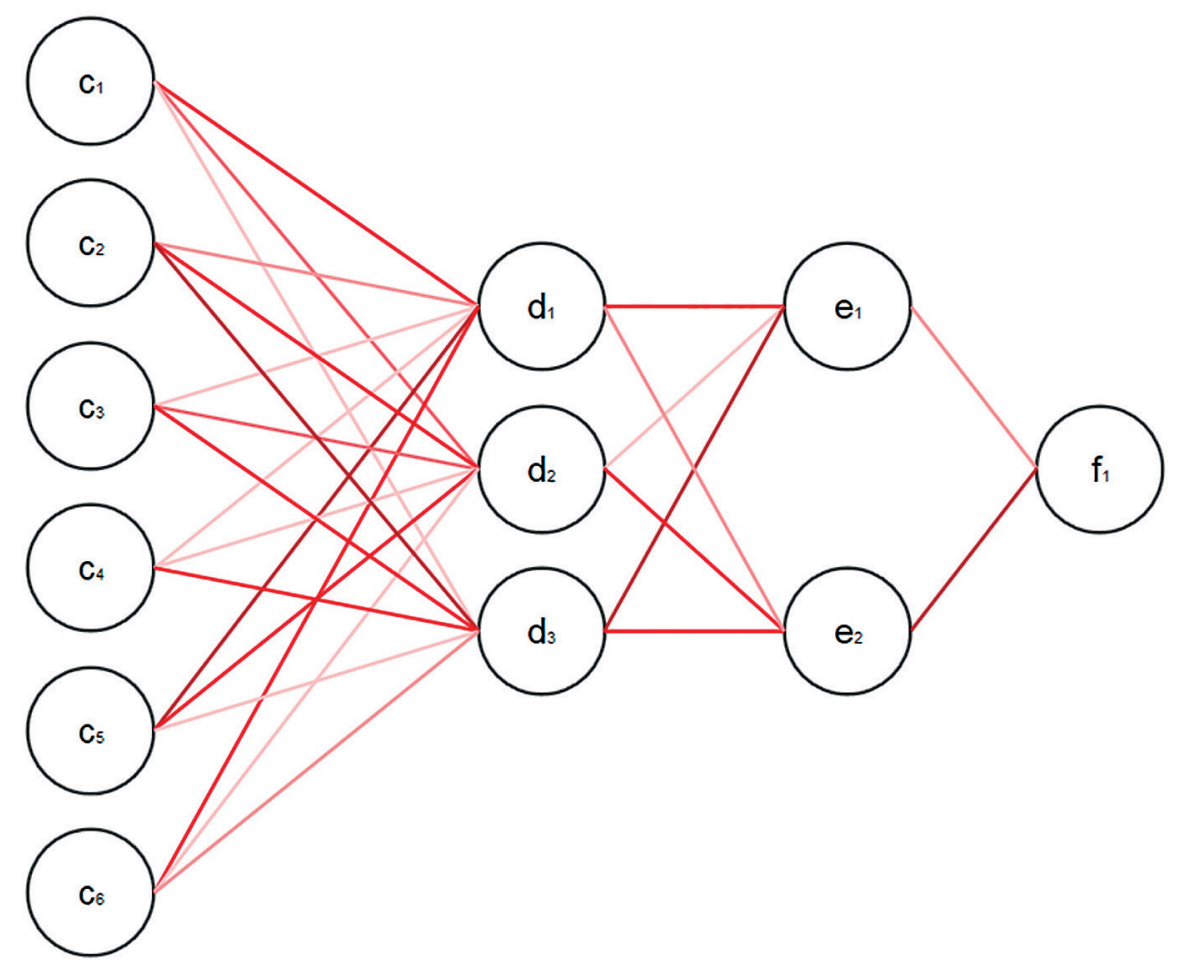

Fig. 2. Schematical visualization of a hypothetical neural network

In the presented in fig. 2 neural network $N$, cells $C_{n}$ respond preferentially to the simplest properties of the stimuli, and the complexity of detected properties rises with successive layers. Color intensity of a connection symbolizes its strength. Properties of the model example for the notion that is associated with 
activation of $f_{1}$ might be then derived by following a path of strongest connections. In possible, the subsequent process of language production, the language network would receive stimulation convergently, from multiple layers of $N$. Note that this figure is an oversimplification which does not include reafference, processing in multiple parallel overlapping networks (i.e. emotional) and several other mechanisms. Separation of any singular network from the brain requires artificial creation of crisp boundaries, whereas in reality they are fuzzy.

\section{Vagueness}

There are two neuroscientific concepts critical to the understanding of vagueness and sorites argumentation, namely all-or-none law and just noticeable difference (JND) principle. However, these concepts originate from different levels of description of cognitive processes - one from cellular neurobiology while the other from psychophysics.

All-or-none law states that all action potentials generated by neurons are approximately of the same size (expressed as a value of cellular membrane depolarization) and do not convey information concerning the magnitude of the stimulus that evoked them. Instead, strength of any stimulus might be coded as a frequency of action potentials. It follows, since there is no gradation in singular responses of a cell, that there is a sensory threshold of a stimulus strength at which a cell will generate an action potential $50 \%$ of the time. The value of that threshold varies depending on a type of receptor and its history of excitation, since repeated exposition to stimuli changes sensory threshold through sensitization. Therefore, any subliminal stimulus does not provide any information for the network to process. This constitutes the most elementary limitation of human cognition in relation to the sorites argument.

One of the earliest laws of psychophysics concerns the smallest difference between two stimuli that people are able to detect. Weber-Fechner law states that JND value is a constant ratio of value of the preceding stimulus. In other words, the amount that is required to be added to the stimulus, in order for the change to be detectable, is a set fraction of that stimulus $\left(\frac{\Delta I}{I}=k\right.$; where $I$ is the strength of the stimulus, $\Delta I$ is the smallest detectable change in the stimulus strength and $k$ is constant). That law does not hold true for all types of stimuli and is in fact 
only an approximation. However, the general idea of JND relativity encompasses all types of sensory perception. It is easiest to grasp it with the help of an example. Imagine two envelopes and that one of them contains a coin. It is trivial to identify the envelope with a coin, through manual examination of its weight. Yet, if we put a coin inside a shoe it would be impossible to discern its weight from an empty one.

When confronted with these laws, it is clear that sorites argumentation puts our mind not only in an unnatural situation, but also impossible to process on grounds of perception. It forces us to refer to purely formal, mathematical procedure and to infer basing on logical, not perceptual understanding of the insignificant difference. In terms of cognitive psychology, the significant difference starts not on the level of sensory threshold and not even on the level of just noticeable difference, but where neural network architecture put its boundary conceived in the process of linguistic competence acquisition. Furthermore, continuous character of the sorites procedure forces us to acknowledge its conclusion as an absolute by virtue of rules governing stability and dynamicity of cognitive processes. Human's perception is constantly dependent on preceding stimuli and characterized by the attention-driven bias in judgment towards what was previously perceived (serial dependence) (Fischer, Whitney, 2014). Imagine a slightly different setting of sorites procedure, where all considered ambiguous cases would be randomly mixed and always paired with a unambigous one. After collection of responses from a person, experimenters could sort them back again in the original way. Emerging distribution of responses would reveal the penumbra, while decisions concerning membership to both cases $A$ and $\sim A$ could be described as fuzzy sets. Then, the examination of neurobiological correlates of decisions taken deep in the penumbra would partially reveal variables that influence judgment in ambiguous situations. They would definitely cover individual differences in properties of neuronal noise, excitability and most importantly, an interpretation of situational context which would i.e. influence people's tendency to equalize the number of $A$ and $\sim A$ answers. Note that heavy influence of a context on any categorization is definitely part of an adaptive behavior, considering that in an environment different mistakes carry different costs with them. Thus, in the end a decision is always made, and can always be changed, making vagueness a problem of linguistics and mathematical interpretation of crisp sets containing notion designates, not of the perception or language production. 


\section{Conclusion}

Theories of logic in their reasoning typically do not pertain to empirical studies. However, if they encompass mental processes, this lack of grounding in principles of cognitive psychology and neurobiology prevents any theory from being truly complete. The contrary can be seen on the example of linguistic competence where processes described on an epiphenomenal level share their structure with underlying biological counterparts. Concepts of sufficient similarity, model examples and partiality of an expression content, among others, are possible to be better explained through the addition of principles that describe the functioning of neural networks.

\section{References}

Atlan, H., Cohen, I.R. (1998). Immune information, self-organization and meaning. International Immunology 10 (6): 711-717.

Bedny, M., Caramazza, A. (2011). Perception, action, and word meanings in the human brain: The case from action verbs. Annals of the New York Academy of Sciences 1224 (1): 81-95.

Binder, J.R., Desai, R.H. (2011). The neurobiology of semantic memory. Trends in cognitive sciences 15 (11): 527-536.

Carlson, T.A., Simmons, R.A., Kriegeskorte, N., Slevc, L.R. (2014). The emergence of semantic meaning in the ventral temporal pathway. Journal of cognitive neuroscience 26 (1): 120-131.

Catani, M., Jones, D.K. (2005). Perisylvian language networks of the human brain. Annals of neurology 57 (1): 8-16.

Chatterjee, A. (2010). Disembodying cognition. Language and cognition 2 (1): 79-116.

Duch, W., Dobosz, K. (2011). Visualization for understanding of neurodynamical systems. Cognitive Neurodynamics 5 (2): 145-160.

Fingelkurts, A.A., Fingelkurts, A.A. (2001). Operational architectonics of the human brain biopotential field: Towards solving the mind-brain problem. Brain and Mind 2 (3): 261-296.

Fischer, J., Whitney, D. (2014). Serial dependence in visual perception. Nature neuroscience 17: 738-743.

Foo, N., Low, B.T. (2008). A Note on Prototypes, Convexity and Fuzzy Sets. Studia Logica 90 (1): 125-137.

Foxe, J.J., Schroeder, C.E. (2005). The case for feedforward multisensory convergence during early cortical processing. Neuroreport 16 (5): 419-423.

Freeman, W.J., (1998). The neurobiology of multimodal sensory integration. Integrative physiological and behavioral science 33 (2): 124-129. 
Freeman, W.J. (2004). How and why brains create meaning from sensory information. International journal of bifurcation and chaos 14 (2): 515-530.

Hart, S.J., Green, S.R., Casp, M., Belger, A. (2010). Emotional priming effects during Stroop task performance. Neuroimage 49 (3): 2662-2670.

Hauk, O., Johnsrude, I., Pulvermüller, F. (2004). Somatotopic representation of action words in human motor and premotor cortex. Neuron 41 (2): 301-307.

Łukowski, P. (2015). Two procedures expanding a linguistic competence. In: P. Łukowski, A. Gemel, B. Żukowski (eds.), Cognition, Meaning and Action. Lodz-Lund Studies in Cognitive Science. Łódź: Wydawnictwo Uniwersytetu Łódzkiego.

Martin, A. (2007). The representation of object concepts in the brain. Annual Reviews Psychology 58: 25-45.

McNamara, T.P. (2005). Semantic priming: Perspectives from memory and word recognition. New York: Psychology Press.

Owen, A.M., Coleman, M.R., Boly, M., Davis, M.H., Laureys, S., Pickard, J.D. (2006). Detecting awareness in the vegetative state. Science 313 (5792): 1402.

Panizza, D. (2012). Formal neurosemantics. Logic, meaning and composition in the brain. Journal of Neurolinguistics 25 (5): 460-488.

Price, C.J. (1998). The functional anatomy of word comprehension and production. Trends in cognitive sciences 2 (8): 281-288.

Pulvermüller, F. (2012). Meaning and the brain: The neurosemantics of referential, interactive, and combinatorial knowledge. Journal of Neurolinguistics 25 (5): 423-459.

Raichle, M.E. (2010). Two views of brain function. Trends in cognitive sciences 14 (4): $180-190$.

Tsumoto, T. (1992). Long-term potentiation and long-term depression in the neocortex. Progress in neurobiology 39 (2): 209-228.

Zeki, S., Romaya, J.P., Benincasa, D.M., Atiyah, M.F. (2014). The experience of mathematical beauty and its neural correlates. Frontiers in human neuroscience 8: 1-12. 